\title{
The idea of fuzzy logic usage in a sheet-based FMEA analysis of mechanical systems
}

\author{
Grzegorz Filo ${ }^{1, *}$, Joanna Fabiś-Domagała ${ }^{1}$, Mariusz Domagała ${ }^{1}$, Edward Lisowski ${ }^{1}$, and \\ Hassan Momeni ${ }^{2}$ \\ ${ }^{1}$ Cracow University of Technology, Faculty of Mechanical Engineering, Jana Pawła II 37, \\ 31-864 Cracow, Poland \\ ${ }^{2}$ Western Norway University of Applied Sciences, Inndalsveien 28, 5063 Bergen, Norway
}

\begin{abstract}
The main purpose of the work which was carried out and is presented in this paper was to examine the possibility of using fuzzy logic inference for conducting a risk analysis with the help of a sheet-based Failure Mode and Effects Analysis method (FMEA). At the beginning, the main features of the analysed method were presented, with particular emphasis put on the Risk, Priority and Number parameters. Then, a proposal has been made which suggests using Matlab Fuzzy Logic Toolbox package in order to convert the factors into the form of fuzzy sets and to define rules for fuzzy inference process has been made. Finally, the created fuzzy logic model was used to present an example analysis of a turbocharger failure in the fuzzified form.
\end{abstract}

\section{Introduction}

Today, in engineering design, a lot of emphasis in engineering design is put on the reliability of the product. One of the methods used for this purpose is Failure Mode and Effects Analysis (FMEA). It consists in an analysis of the causes and effects of defects, and became one of the fundamental methods used to improve the quality of products, processes and services. It was developed in the United States as a military procedure MIL-P 1629 [1]. Then, in the 1960s, it was used by NASA in the Apollo Saturn space flight project. The flight to the moon succeeded, therefore the method was used in other industries, especially in nuclear and aerospace industry. Since 1970 FMEA has become more and more popular in the automotive industry, e.g. Ford Motor Company used FMEA to analyse cars in terms of their safety for the user and the fulfilment of legal requirements. Suppliers of parts and subassemblies for the production of Ford cars were obliged to use the method in accordance with the ISO/TS 16949 standard previously known as QS-9000. Currently, FMEA has a very wide usage and can be applied in many fields, including medicine, metallurgy, food services, engineering, and even public administration. The analysis can be carried out in accordance with the EN 60812:2006 standard [2], and it has been included into the ISO standard [3]. The main goal is to consistently and systematically identify potential defects in products or processes and then eliminate or minimize the risks associated with them. The

\footnotetext{
*Corresponding author: filo@mech.pk.edu.pl
} 
greatest benefits are brought when the method is used at the initial stage of concept and design, because in this case the costs of defect removal is the lowest.

Several variants of the FMEA method can be distinguished. However, due to the preparation process, the way of conducting and presenting the obtained results, it can be divided into a sheet-based analysis (classical) and a matrix-based analysis (FFDM) [4]. A state of the art summary of the classical FMEA method was published in the form of a review article by Spreafico et al. in 2017 [5]. The review consists in analysing a representative pool of over 200 scientific papers and over 100 patents in order to have an overview of the evolution of the method. It shows a large number of problems which have already been solved using FMEA. However, the interest in the method is still growing. One of the ways of its development is combining the classic FMEA analysis with the fuzzy set theory. A novel modelling approach based on the intuitionistic fuzzy approach was proposed by Tooranlo and Ayatollah in [6]. The approach offers advantages over earlier models since it accounts for degrees of uncertainty in relationships among various criteria or options, specifically when relations cannot be expressed in definite numbers, and provides a tool to evaluate failure modes in the case of insufficient data. Another methodology, which integrates the concepts of similarity value measure of fuzzy numbers and the theory of possibility was developed by Mandal and Maiti [7]. New proposals for the application of fuzzy logic met with great interest of scientific centres, which resulted in creation of many hybrid FMEA methods and models in the fuzzy environment, such as extended MULTIMOORA or AHP, applied e.g. by Fattahi [8] and Hu-Chen [9].

The developed methods have already been used in a number of practical applications. Considering the fields related to mechanical engineering, most of the articles are devoted to the analysis of drives and control systems. Zaifang [10] proposed a fuzzy RPN-based method that integrates the weighted least square method, the method of imprecision and partial ranking method to generate more accurate fuzzy RPNs and ensure robustness against the uncertainty. The method was demonstrated by the design example of a new horizontal directional drilling machine. Xu [11] presented a fuzzy logic based method for the FMEA analysis of a diesel engine turbocharger system, while Renjith [12] carried out fuzzy FMECA (failure mode effect and criticality analysis) of a liquid natural gas storage facility, and Yazdi [13] used a similar FDFMEA method (Fuzzy Developed FMEA) for an aircraft landing system. With regard to the presented state of art, this article concerns creation of a sheet-based FMEA model using fuzzy logic in Matlab environment. The model was used to demonstrate the analysis of an example turbocharger failure.

\section{Fuzzy model of a sheet-based FMEA method}

In general, risk assessment in the sheet-based FMEA method is carried out using a risk priority number $(R P N)$, which can be estimated on the basis of three factors: Occurrence $(O)$, Severity $(S)$ and Detection $(D)$. In the conventional approach, the value of the $R P N$ is a simple product of all three factors: $R P N=O \cdot S \cdot D$. The value of each factor is an integer number of range 1 to 10 . The analysis is usually carried out in three stages [14]: preparation of the analysis, conducting the analysis, as well as introduction and supervision of improvement activities. The diagram of a complete procedure is shown in Fig. 1.

The application of fuzzy logic requires transformation of integer numbers (crisp values) into degrees of fuzzy set memberships (fuzzy values). For this purpose, first fuzzy sets of all input parameters $(O, S, D)$ as well as outputs $(R P N)$ must be defined. The detailed process of a fuzzy logic model creation is presented e.g. by Piegat [15], while its practical application illustrated on the example of a sterilization unit risk analysis is shown by Dağsuyu [16]. Crisp values of the factors with verbal description and names adopted for defining fuzzy sets are given in Table 1. 


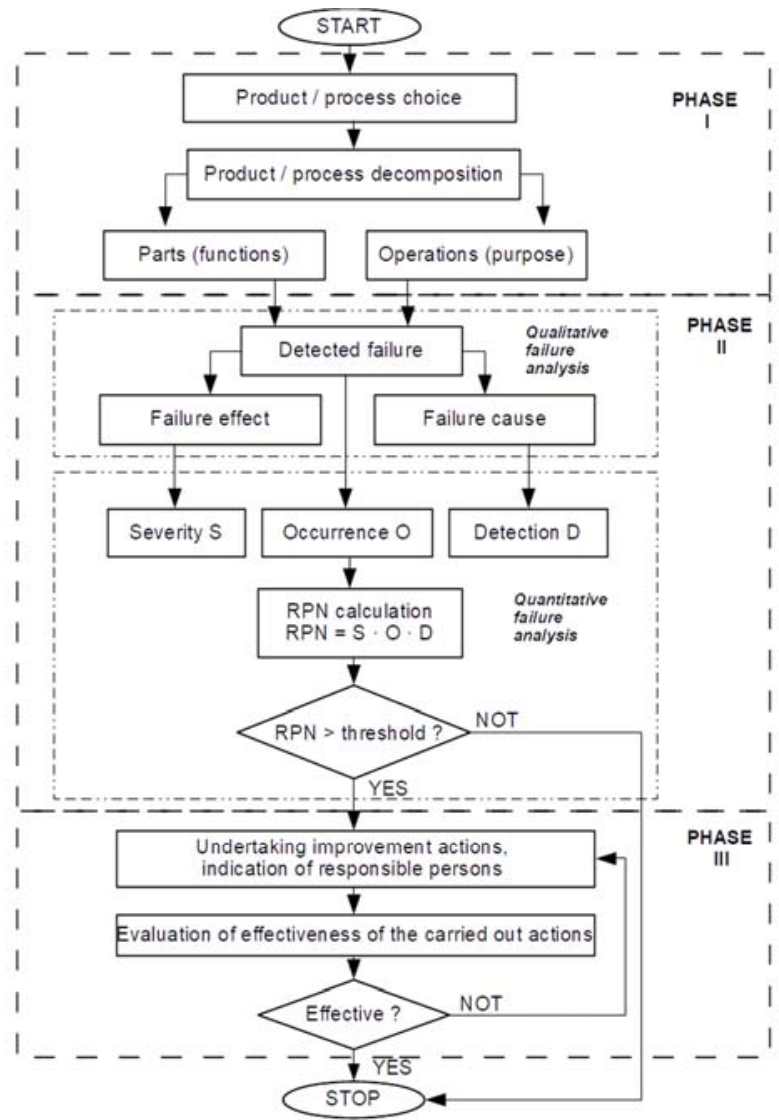

Fig. 1. Process of a conventional FMEA analysis.

Table 1. Crisp values and names of fuzzy sets defined for the $O, S, D$ factors.

\begin{tabular}{|c|c|c|c|c|c|c|}
\hline \multirow{3}{*}{$\begin{array}{l}\text { Crisp } \\
\text { value }\end{array}$} & \multicolumn{6}{|c|}{ Factor } \\
\hline & \multicolumn{2}{|c|}{ Occurrence $O$} & \multicolumn{2}{|c|}{ Severity $S$} & \multicolumn{2}{|c|}{ Detection D } \\
\hline & verbal & $\begin{array}{c}\text { fuzzy } \\
\text { set }\end{array}$ & verbal & $\begin{array}{c}\text { fuzzy } \\
\text { set }\end{array}$ & verbal & $\begin{array}{c}\text { fuzzy } \\
\text { set }\end{array}$ \\
\hline 1 & Absent & VL & Unnoticeable & $\mathrm{VL}$ & Very high & $\mathrm{VH}$ \\
\hline 2,3 & Rare & $\mathrm{L}$ & Small meaning & $\mathrm{L}$ & High & $\mathrm{H}$ \\
\hline $4,5,6$ & Sometimes & $M$ & Moderate & M & Medium & $\mathrm{M}$ \\
\hline 7,8 & Often & $\mathrm{H}$ & Serious & $\mathrm{H}$ & Low & $\mathrm{L}$ \\
\hline 9,10 & Very often & $\mathrm{VH}$ & Critical & VH & Very low & VL \\
\hline
\end{tabular}

According to Table 1, the following fuzzy sets were defined for each input factor: VL (very low), L (low), M (medium), H (high) and VH (very high). Such unification of the fuzzy set names significantly increases consistency of the model. Due to the fact, that the cores (subsets with the maximum value of the membership functions) have the form of intervals in most fuzzy sets (e.g. the core of the fuzzy set L of Occurrence factor is in the range from 2 to 3 ), instead of the commonly used linear-type membership functions [6, 7 , $10,14]$, the Gauss-type functions were applied in this model, similarly to [11]. The scheme of the model created in Anfis editor is presented in Fig. 2. The diagram of fuzzy sets defined for the Occurrence $O$ input factor is shown in Fig. 3, while the diagram of Detection $D$ is presented in Fig. 4. The Severity diagram is omitted, since it has the similar form to Occurrence. The $R P N$ failure classification factor has the crisp value from the range of [1, 
1000]. It was divided into seven fuzzy sets, using a similar membership function set as introduced in [12], respectively: VL (very low, core less than 10), ML (medium low, core between 20 and 30), LM (low medium, core 90-110), MM (medium medium, core 180220), HM (high medium, core 330-370), MH (medium high, core 460-540) and VH (very high, core 750-1000). The diagram of fuzzy sets defined for the $R P N$ factor is shown in Fig. 5.

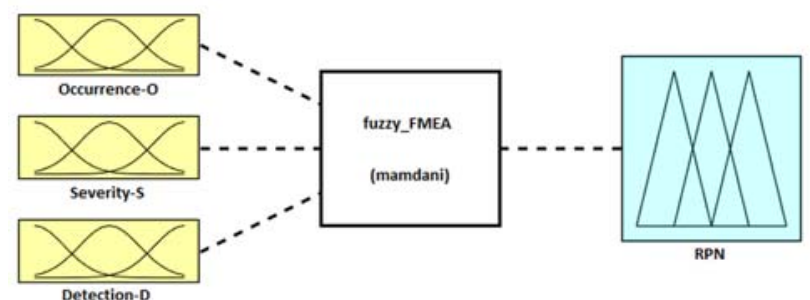

Fig. 2. Scheme of fuzzy FMEA model created with Matlab Anfis editor.
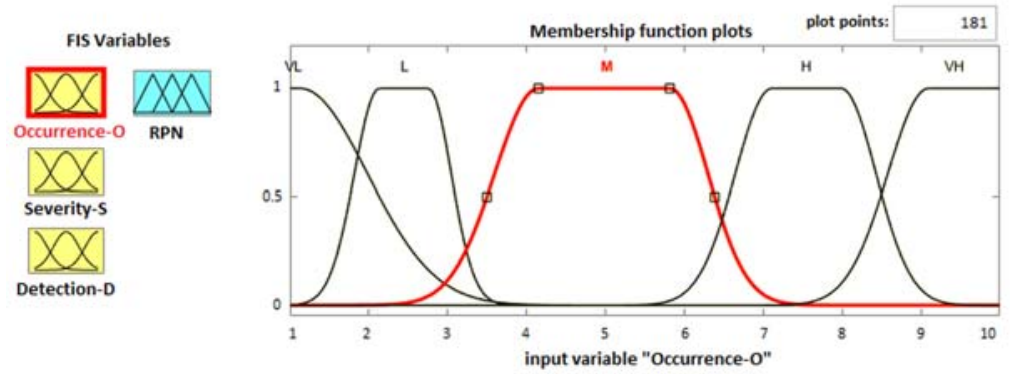

Fig. 3. Fuzzy sets defined for Occurrence factor using Gauss-type membership functions.
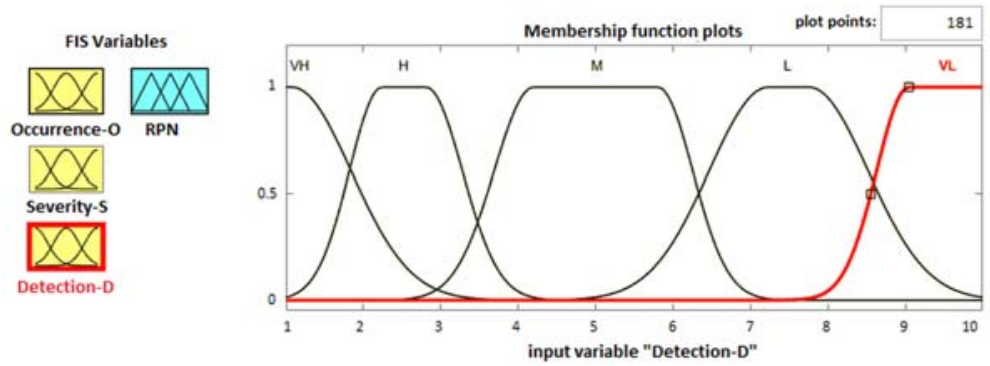

Fig. 4. Fuzzy sets defined for Detection factor using Gauss-type membership functions.
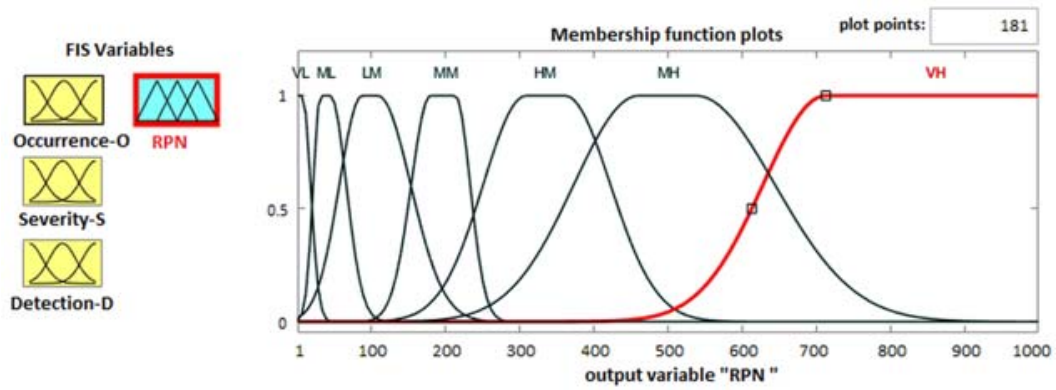

Fig. 5. Fuzzy sets defined for $R P N$ factor using Gauss-type membership functions. 
In the next step, fuzzy mathematical operators as well as the defuzzification method required by the inference process were determined. Based on the analysis of previous research, the following parameters have been selected: fuzzy product operator: prod, fuzzy sum operator: probor, the defuzzification method: Centroid. Finally, a database of rules which represent connections between the fuzzy sets of input factors and RPNs has been created. The database consists of more than 100 rules in the form of if-then statements.

\section{Practical application of created fuzzy FMEA model}

As a practical example will be presented application of the proposed fuzzy model in the analysis of a selected turbocharger defect, resulting in decrease in engine power [17]. The visible symptoms of the fault were noise and vibrations, hence the following factor values were assigned: $S=7, O=8, D=4$. The fuzzy system gives in this case the $R P N$ value of 500 (Fig. 6), which means the problem is considered to be serious (the most activated fuzzy set of RPN is $\mathrm{VH})$.

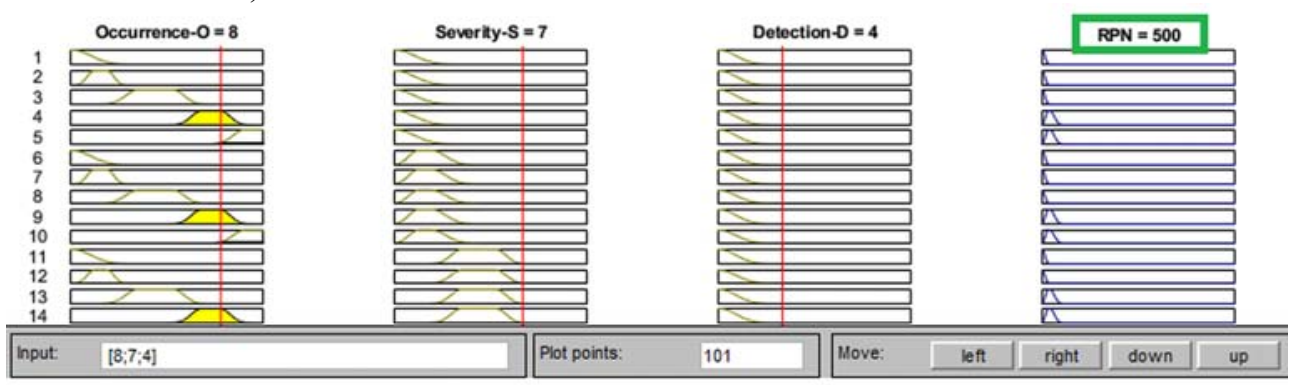

Fig. 6. Fuzzy FMEA system output for $\mathrm{S}=7, \mathrm{O}=8, \mathrm{D}=4$.

After the repair, which consisted of replacing the air filter, values of the Severity and Occurrence factors changed respectively to: $S=5, O=6$. The $R P N$ generated by the fuzzy model in this case has been reduced to RPN $=190$, which means the highest activation of the LM and MM fuzzy sets.

\section{Conclusion}

This paper has presented a proposal to use Matlab Fuzzy Logic Toolbox to carry out a sheet-based FMEA analysis. A fuzzy model was created in Anfis editor and then was used to analyse a selected turbocharger defect. The obtained $R P N$ values are higher than those from the classical FMEA method [17]: $\mathrm{RPN}=224$, and $\mathrm{RPN}=120$, before and after the repair, respectively. However, in both cases the $R P N$ value was approximately halved.

Regardless of the specific described application, similar approach may be used to manage the risk in interdisciplinary long-term research like e.g. biotechnological investigation [18, 19] or materials science industry-oriented investigations [20-32]. Similar problems are also met in strict management area of the industry and the academia e.g. [3337]. Another related issues worth investigating more closely are studies on non-classically estimated fuzzy uncertainty e.g. finite element combined with time series [38, 39], a fixedeffect approach [40] or a bootstrap simulations [41, 42]. Further investigations intend finetuning of the created model parameters and using it to analyse more complex systems. 


\section{References}

1. USA military standard, Procedure for Performing a Failure Mode, Effects and Criticality Analysis (MIL-P 1629, USA, 1949)

2. EN 60812:2006 standard, Analysis techniques for system reliability. Procedure for failure mode and effects analysis (FMEA), (2006)

3. ISO 9001:2015 standard, Quality Management Systems - Revision, (2015)

4. J. Fabiś-Domagała, Tribologia, 2, 33-38 (2017)

5. C. Spreafico, D. Russo, C. Rizzi, Comp. Science Rev., 25, 19-28 (2017)

6. H. S. Tooranlo, A. Ayatollah, Appl. Soft Comput. 49, 238-247 (2016)

7. S. Mandal, J. Maiti, Expert Syst. Appl. 41, 3527-3537 (2014)

8. R. Fattahi, M. Khalilzadeh, Saf. Sci., 102, 290-300 (2018)

9. L. Hu-Chen, F. Xiao-Jun, L. Ping, C. Yi-Zeng, Eng. Appl. Artif. Intell., 34, 168-177 (2014)

10. Z. Zaifang, C. Xuening, Expert Syst. Appl., 38, 206-214, (2011)

11. K. Xu, L.C. Tang, M. Xie, S.L. Ho, M.L. Zhu, Reliab. Eng. Syst. Saf. 75, 17-29 (2002)

12. V.R. Renjith, M.J. Kalathil, P. H. Kumara, D. Madhavan, J. Loss Prev. Process Ind., (2018)

13. M. Yazdi, S. Daneshvar, H. Setareh, An extension to Fuzzy Developed Failure Mode and Effects Analysis (FDFMEA) application for aircraft landing system (Safety Science, 98, 2017)

14. A. Hamrol, Management and quality engineering (PWN, Warszawa, Poland, 2017)

15. A. Piegat, Modeling and fuzzy control (AOW EXIT, Warszawa, Poland, 1999)

16. C. Dağsuyu, E. Göçmen, M. Narl,A. Kokangül, Comput. Ind. Eng., 101, 286-294 (2016)

17. J. Fabiś-Domagała, Technical Trans., 111(4), 11-17 (2014)

18. E. Skrzypczak-Pietraszek, J. Pietraszek, Chem. Biodivers., 11, 562 (2014)

19. E. Skrzypczak-Pietraszek, I. Kwiecien, A. Goldyn, J. Pietraszek, Phytochem. Lett., 20, 443 (2017)

20. T. Pieczonka, J. Kazior, A. Tiziani, A. Molinari, J. Mater. Process. Technol., 64, 327 (1997)

21. A. Dudek, C. Kolana, Solid State Phenom., 165, 25 (2010)

22. M. Ulewicz, U. Lesinska, M. Bochenska, Physicochem. Probl. Mi., 44245 (2010)

23. D. Klimecka-Tatar, S. Borkowski, P. Sygut, Arch. Metall. Mater., 60, 735 (2015)

24. M. Ulewicz, E. Radzyminska-Lenarcik, Physicochem. Probl. Mi., 46, 119 (2011)

25. D. Klimecka-Tatar, G. Pawlowska, M. Sozanska, Arch. Metall. Mater., 60153 (2015)

26. R. Ulewicz, P. Szataniak, F. Novy, Proc. $23^{\text {rd }}$ International Conference on Metallurgy and Materials (Brno, 2014)

27. P. Szataniak, F. Novy, R. Ulewicz, Proc. $23^{\text {rd }}$ International Conference on Metallurgy and Materials (Brno, Czech Republic, 2014)

28. A. Szczotok, Materialwiss. Werkstofftech., 46, 320 (2015)

29. A. Szczotok, J. Nawrocki, J. Pietraszek, Arch. Metall. Mater., 62, 587 (2017)

30. R. Dwornicka, N. Radek, M. Krawczyk, P. Osocha, J. Pobedza, Proc. 26th Int. Conf. on Metall. and Mater. - METAL (Brno, Czech Republic, 2017) 
31. J. Pobedza, A. Sobczyk, Key. Eng. Mater., 542, 143 (2013)

32. M. Niemczewska-Wojcik, W. Pielzoszewski, Arch. Civ. Mech. Eng., 17, 344 (2017)

33. Kozien, E., Proc. 20 th Int. Sci. Conf. on Economic and Social Dev. (ESD) (Prague, Czech Republic, 2017)

34. Kozien, E., Kozien, A., Proc. 23 ${ }^{\text {th }}$ Int. Sci. Conf. on Economic and Social Dev. (ESD) (Madrid, Spain, 2017)

35. Kozien, E., Kozien, M.S., Proc. $26^{\text {th }}$ Int. Sci. Conf. on Economic and Social Dev. - Building Resilient Society (ESD) (Zagreb, Croatia, 2017)

36. Kozien, E., Kozien, A., Proc. $26^{\text {th }}$ Int. Sci. Conf. on Economic and Social Dev. - Building Resilient Society (ESD) (Zagreb, Croatia, 2017)

37. E. Kozień, Proc. $31^{\text {st }}$ Int. Sci. Conf. on Economic and Social Dev. - Legal Challenges of Modern World (ESD) (Split, Croatia, 2018)

38. I. Dominik, J. Kwasniewski, K. Lalik, R. Dwornicka, Proc. $32^{\text {nd }}$ Chinese Control Conf. (CCC) (Xian, China, 2013)

39. E. Augustyn, M.S. Kozien, Acta Phys. Pol. A, 125, A164 (2014)

40. J. Pietraszek, A. Gądek-Moszczak, T. Torunski, Adv. Mat. Res.-Switz., 874, 139 (2014)

41. J. Pietraszek, A. Gadek-Moszczak, Solid State Phenom., 197, 162 (2013)

42. J. Pietraszek, M. Kolomycki, A. Szczotok, R. Dwornicka, Lect. Notes. Artif. Int., 9875, 260 (2014) 